\title{
The evolution of Massarineae with Longipedicellataceae fam. nov.
}

\section{Phukhamsakda $\mathrm{C}^{1,3,5}$, Hongsanan $\mathrm{S}^{1,2,3^{*}}$, Ryberg $\mathbf{M}^{4}$, Ariyawansa $\mathrm{HA}^{6}$, Chomnunti $\mathrm{P}^{3,5}$, Bahkali AH ${ }^{7}$, Hyde $\mathrm{KD}^{1,3,6,7}$}

${ }^{1}$ Key Laboratory for Plant Diversity and Biogeography of East Asia, Kunming Institute of Botany, Chinese Academy of Science, Kunming 650201, Yunnan, China

${ }^{2}$ World Agroforestry Centre, East and Central Asia, Kunming 650201, Yunnan, People's Republic of China

${ }^{3}$ Center of Excellence in Fungal Research, Mae Fah Luang University, Chiang Rai 57100, Thailand

${ }^{4}$ Department of Organismal Biology, Uppsala University, Norbyvägen 18D, 752 36, Uppsala, Sweden

${ }^{5}$ School of Science, Mae Fah Luang University, Chiang Rai 57100, Thailand

${ }^{6}$ Guizhou Key Laboratory of Agricultural Biotechnology, Guizhou, Academy of Agricultural Sciences, Guiyang 550006, Guizhou, China

${ }^{7}$ Department of Botany and Microbiology, King Saudi University, Riyadh, Saudi Arabia

Phukhamsakda C, Hongsanan S, Ryberg M, Ariyawansa HA, Chomnunti P, Bahkali AH, Hyde KD 2016 - The evolution of Massarineae with Longipedicellataceae fam. nov. Mycosphere 7 (11), 1713-1731, Doi 10.5943/mycosphere/7/11/7

\begin{abstract}
Massarineae is a suborder of Pleosporales, the latter being the largest order in Dothideomycetes. Massarineae comprises 14 families and six taxa of uncertain placement. In this study, we introduce an additional new family, Longipedicellataceae in Massarineae, which accommodates the genera Longipedicellata and Pseudoxylomyces. The family inhabits submerged culms of plants in freshwater habitats. The family can be distinguished by its very long pedicellate asci and chlamydospore-like structures, which are produced in culture. A LSU, SSU, and RPB2 dataset from representative strains used in our phylogenetic analyses shows the separation of Longipedicellataceae from the other families of Massarineae. In addition, divergence times of families in Massarineae were estimated using a molecular clock methodology. We used an Eocene fossil of Margaretbarromyces dictyosporus to estimate dates in Pleosporales with emphasis on Massarineae. In this study, the crown of Pleosporales is dated to the late Triassic (211 Mya), while the suborder Massarineae is dated to the Cretaceous (130 Mya) and family Longipedicellataceae is dated to Eocene (56 Mya).
\end{abstract}

Key words - BEAST - Chlamydospores - Fossil fungi - freshwater fungi - Margaretbarromyces dictyosporus - Pleosporales

\section{Introduction}

Pleosporales is the largest order in the class Dothideomycetes and has thus been the focus of recent research (Zhang et al. 2012, Hyde et al. 2013, Wijayawardene et al. 2014, Ariyawansa et al. 2015). Taxa from this order occur in terrestrial, marine and freshwater habitats and have a worldwide distribution (Campbell et al. 2006, Kruys \& Wedin 2009, Zhang et al. 2009, Tanaka et al. 2015, Jones et al. 2015, Ertz et al. 2015). The order comprises two main suborders, Massarineae and Pleosporineae, which is supported in morphological and phylogenetic studies (Zhang et al. 2012, Ariyawansa et al. 2015, Hyde et al. 2016). The suborder Massarineae was established by Barr 
(1979) to accommodate Arthopyreniaceae Watson, Massarinaceae Munk (family typified), and Pleomassariaceae Barr. Massarineae was characterized by depressed ascomata, with ostioles and thick peridia at the sides (Barr 1979). However, modern classifications using phylogenetic analysis together with morphological circumscription have demonstrated that Arthopyreniaceae and Pleomassariaceae are distinct from Massarinaceae. Thus, they were excluded from the suborder Massarineae (Schoch et al. 2009, Zhang et al. 2012, Hyde et al. 2013, Liu et al. 2014).

The suborder Massarineae currently includes 12 families: Bambusicolaceae, Dictyosporiaceae, Didymosphaeriaceae, Lentitheciaceae, Latoruaceae, Macrodiplodiopsidaceae, Massarinaceae, Morosphaeriaceae, Parabambusicolaceae, Periconiaceae, Sulcatisporaceae, and Trematosphaeriaceae (Zhang et al. 2012, Hyde et al. 2016). Mapook et al. (2016) placed Phaeodimeriella (Pseudoperisporiaceae) in the suborder Massarineae based on their phylogenetic analysis. The placements of Asteromassaria pulchra (Harkness) Shoemaker \& P.M. LeClair, Inflatispora pseudostromatica Zhang, J. Fournier \& K.D. Hyde Monodictys capensis Sinclair, S. Boshoff \& A. Eicker, Bactrodesmium cubense (Castañeda \& Arnold) Zucconi \& D. Lunghini, Pseudoxylomyces elegans Tanaka \& K. Hirayama and Fuscostagonospora sasae Tanaka \& K. Hirayama are uncertain, thus they are included as species and genera incertae sedis within Massarineae (Boonmee et al. 2016). Zhang et al. (2016) introduced Longipedicellata aptrootii (Hyde \& Wong) Zhang, K.D. Hyde \& J.K. Liu to accommodate Didymella aptrootii, a species collected from submerged bamboo in freshwater (Hyde \& Wong 1999) and assigned it to Bambusicolaceae. However, our phylogenetic analysis indicates that the species is closely related to Pseudoxylomyces elegans (Goh, W.H. Ho, K.D. Hyde \& K.M. Tsui) Tanaka \& K. Hirayama, where they form a distinct clade.

Divergence time estimates using molecular clock methodologies have been developed for the fungi (Berbee \& Taylor 1993, Li et al. 2005, Taylor \& Berbee 2006, Vijaykrishna et al. 2006). Various pleosporalean fossils have been discovered and can be compared to extant genera, introducing new fossil for dating in molecular clocks (Mindell 2007, Taylor et al. 2009, 2015). In the present study, we (1) estimate divergent times for families of the suborder Massarineae (Pleosporales) using a molecular clock methodology based on LSU, SSU, and RPB2 sequence data and (2) introduce Longipedicellataceae fam. nov. based on phylogenetic and evolutionary evidence, with includes the genera Longipedicellata and Pseudoxylomyces.

\section{Material \& Methods}

\section{Sample collection, morphological study and isolation}

Fresh materials were collected from submerged stems of Bambusodeae in Chiang Rai, Thailand during 2015 and recollected in 2016. Fresh specimens were kept in plastic Ziploc bags with a small amount of water and returned to the laboratory. Pure cultures were established from single ascospores on $2 \%$ potato dextrose agar (PDA; $39 \mathrm{~g} / \mathrm{L}$ Difco potato dextrose in distilled water) and malt extract agar (MEA; $62 \mathrm{~g} / \mathrm{L}$ Criterion in distilled water) as described in Chomnunti et al. (2014). Cultures were incubated at $25^{\circ} \mathrm{C}$ for up to 8 weeks. Type specimens were deposited in Mae Fah Luang University (MFLU) herbarium. Ex-type living cultures were deposited at the Mae Fah Luang Culture Collection (MFLUCC). Faces of fungi numbers and Index Fungorum numbers are provided (Jayasiri et al. 2015, Index Fungorum 2016). Samples were examined under a Nikon ECLIPSE 80i compound microscope and photographed with a Canon 600D digital camera fitted to the microscope. Measurements were made using Tarosoft $(\mathrm{R})$ Image Frame Work program and photo-plate were made by using Adobe Photoshop CS6 Extended version 10.0 software (Adobe Systems, United States).

\section{DNA extraction, amplification and sequencing}

DNA was extracted from mycelium with Biospin Fungus Genomic DNA Extraction Kit (BioFlux ${ }^{\circledR}$ ) (Hangzhou, P. R. China); following the manufacturer's protocol. PCR amplification was carried out using primers LROR/LR5 for the nuclear ribosomal large subunit 28S rRNA gene (LSU), NS1/NS4 for the nuclear ribosomal small subunit 18S rRNA gene (SSU), and RPB2- 
5f2/RPB2-7cr for RNA polymerase subunit II gene region (RPB2) (Vilgalys \& Hester 1990, White et al. 1990, Carbone \& Kohn 1999). Primer sequences are available at the WASABI database at the AFTOL website (aftol.org). Amplification reactions for LSU and SSU were performed according to Phukhamsakda et al. (2015). The PCR thermal cycle program for RPB2 was set for denaturation at $95{ }^{\circ} \mathrm{C}$ for $5 \mathrm{~min}$, followed by 35 cycles of denaturation at $95{ }^{\circ} \mathrm{C}$ for $1 \mathrm{~min}$, annealing at $52{ }^{\circ} \mathrm{C}$ for 2 min and extension at $72{ }^{\circ} \mathrm{C}$ for $2 \mathrm{~min}$, with a final extension step at $72{ }^{\circ} \mathrm{C}$ for $10 \mathrm{~min}$. DNA extracted and PCR proliferation products were checked on 1\% Agarose gels with added $6 \mu \mathrm{l}$ in 100 $\mathrm{ml}$ of $4 \mathrm{~S}$ green dyes. Purified PCR products and the sequencing were performed by Shanghai Sangon Biological Engineering Technology \& Services Co. (Shanghai, P.R. China).

\section{Sequence alignment and phylogenetic analysis}

SeqMan v. 7.0.0 (DNASTAR, Madison, WI) was used to assemble consensus sequences. Sequences of closely related strains were retrieved using BLAST searches against GenBank (http://www.ncbi.nlm.nih.gov). We also included the strains from Tanaka et al. (2015), Sharma et al. (2015), Boonmee et al. (2016), Mapook et al. (2016) and Hongsanan et al. (2016) and these are listed in Table 1. Sequences were aligned with MUSCLE in MEGA 6 (Tamura et al. 2013). The alignments were checked visually and improved manually where the ambiguous nucleotides presented by using Bioedit 7.2 (Hall 1999). Leading or trailing gaps exceed from primer binding site were trimmed from the alignments prior to tree building. Maximum likelihood analyses (ML), including 1000 bootstrap replicates, was performed using RAxML (Stamatakis 2014) as implemented in raxmlGUI version v.1.3.1 (Silvestro \& Michalak 2012). The search strategy was set to rapid bootstrapping. The analysis was carried out with the general time reversible (GTR) model for nucleotide substitution and a discrete gamma-distributed with four rate categories (O'meara et al. 2006, Stamatakis et al. 2008, Guindon et al. 2010). The bootstrap replicates were summarized on to the best scoring tree. Maximum likelihood bootstrap values equal or greater than $70 \%$ are given in black below or above each node (Fig. 1).

The best model for different genes partition in the concatenated data set was determined in MrModeltest 2.3 (Nylander 2004) for posterior probability (PP). In our analysis, GTR+I+G model was used for each partition. The posterior probability (PP) distribution (Zhaxybayeva \& Gogarten 2002) was estimated by Markov Chain Monte Carlo sampling (MCMC) in MrBayes v. 3.2.2 (Huelsenbeck \& Ronquist 2001). Four simultaneous Markov chains were run for 10,000,000 generations and trees were sampled every 1000th generation, thus 10,000 trees were obtained. The suitable burn-in phase were determined by traces inspected in Tracer version 1.6 (Rambaut et al. 2014). Based on the tracer analysis, the first 1,000 trees representing $10 \%$ of burn-in phase of the analyses were discarded. While the remaining trees were used for calculating posterior probabilities in the majority rule consensus tree (critical value for the topological convergence diagnostic set to 0.01). Bayesian Posterior Probabilities (PP) equal or greater than 0.90 for the clades in the ML tree are given above each node in black (Fig. 1).

\section{Fossil calibration and Divergence time estimates}

Divergence time estimation was carried out in BEAST 1.8.0 (Drummond et al. 2012). We performed two different dating scenarios. Scenario 1 used a normal posterior distribution for the divergence between Arthoniomycetes and Dothideomycetes and the crown age of Capnodiales. The split between Arthoniomycetes (outgroup) and Dothideomycetes was given a secondary calibration point according to Gueidan et al. (2011), Prieto \& Wedin (2013), Beimforde et al. (2014) and Pérez-Ortega et al. (2016), with a mean of 300 Mya and a standard deviation (SD) of 50 Mya, which give a lower boundary of 382 Mya on the $95 \%$ credibility interval (CI; node 1, Fig. 2). The crown age of Capnodiales was set based on the fossil of Metacapnodiaceae and given a mean of 100 Mya, and SD of 150 Mya, giving a lower boundary of 346 Mya (Gueidan et al. 2011, Prieto \& Wedin 2013, Pérez-Ortega et al. 2016, Hongsanan et al. 2016; node 2, Fig. 2). For scenario 2, we also set a constraint for the Aigialus (Aigialaceae) crown based on the fossil Margaretbarromyces dictyosporus (Mindell et al. 2007, Berbee \& Taylor 2010, Taylor et al. 2015). For this calibration 
we used a gamma distribution, with an offset of 35 Mya based on the minimal ages of amber (Mindell et al. 2007), and a shape of 1.0 and scale of 25, giving a lower boundary of the 95\% CI of 110 Mya (node 3, Fig. 2).

Nucleotide substitution models determined by jModelTest version 2.1.10 (Darriba et al. 2012). The GTR+I+G nucleotide substitution model were applied to LSU, SSU, and RPB2 partitions based on the results from jModelTest 2.1.10. The BEAST run was prepared in BEAUti 1.8.0 (BEAST package). The data partitions were set with unlinked substitution, linked clock models and linked tree. Calibration points were set as described for the two scenarios above. A lognormal distribution was used for the relaxed clock applied for the datasets. The Yule speciation process was used as tree prior, which specifies a constant rate of speciation divergence (Gernhard et al. 2008). The value prior was carried out using mean 1.00 in units of substitutions per site per time unit. The analyses were performed for 100 million generations. Tracer version 1.6 was used to check the effective sample sizes (ESS), assuring values more than 200. To validate the analysis, we performed two independent analyses, sampling parameters every 10,000th generation. Based on the tracer analysis the first 1,000 trees, i.e. $10 \%$, were discarded as burn-in. The remaining trees were combined by using LogCombiner 1.8.0 (BEAST package). The maximum clad creditability (MCC) was calculated in TreeAnnotator 1.8.0 (BEAST package).

The geological time scale in this study were retrieved from the International Commission on Stratigraphy (ICS) website (www.stratigraphy.com). The chronostratigraphy chart version 2016/04 were applied to estimate the geological time scale at the base of Fig. 2.

Phylogenetic trees and data files were visualized in FigTree v. 1.4 (Rambaut \& Drummond 2008). The phylograms with bootstrap values and/or posterior probabilities on the branches are presented in Fig. 1 and 2 by using graphical options available in Adobe Illustrator CS v. 6. All sequences generated in this study were submitted to GenBank.

Table 1 GenBank, culture collection code and accession numbers used in this study.

\begin{tabular}{|c|c|c|c|c|}
\hline \multirow{2}{*}{$\operatorname{Taxon}^{1}$} & \multirow{2}{*}{ Strain number } & \multicolumn{3}{|c|}{ GenBank accession numbers } \\
\hline & & LSU & SSU & RPB2 \\
\hline Aigialus grandis & BCC 20000 & GU479775 & GU479739 & GU479814 \\
\hline Aigialus mangrovei & BCC 33563 & GU479776 & GU479741 & GU479815 \\
\hline Aigialus parvus & BCC 18403 & GU479778 & GU479743 & GU479817 \\
\hline Aigialus rhizophorae & BCC 33572 & GU479780 & GU479745 & GU479819 \\
\hline Aliquandostipite khaoyaiensis & CBS 118232 & GU301796 & NG_016494 & - \\
\hline Alternaria alternata & CBS $916.96^{\mathrm{T}}$ & DQ678082 & KC $\overline{5} 84507$ & KC584375 \\
\hline Amniculicola lignicola & CBS $123094^{T}$ & EF493861 & EF493863 & EF493862 \\
\hline Anteaglonium abbreviatum & ANM 925a & GQ221877 & - & - \\
\hline Anteaglonium globosum & GKML101N & GQ221875 & - & - \\
\hline Antennariella placitae & CBS $124785^{T}$ & GQ303299 & - & - \\
\hline Aquastroma magniostiolata & CBS $139680^{T}$ & $\mathrm{AB} 807510$ & AB797220 & - \\
\hline Aquaticheirospora lignicola & RK-2006a ${ }^{T}$ & AY736378 & AY736377 & - \\
\hline Aquilomyces patris & CBS $135661^{T}$ & KP184041 & KP184077 & - \\
\hline Aquilomyces rebunensis & CBS $139684^{T}$ & AB807542 & AB797252 & - \\
\hline Arthonia dispersa & UPSC2583 & AY571381 & AY571379 & \\
\hline Ascocratera manglicola & CBS 120023 & GU301799 & GU296136 & GU371763 \\
\hline Asteromassaria pulchra & CBS 124082 & GU301800 & GU296137 & GU371772 \\
\hline Bactrodesmium cubense & CBS 680.96 & $\mathrm{AB} 807508$ & AB797218 & - \\
\hline Bambusicola bambusae & MFLUCC 11-0614 ${ }^{\mathrm{T}}$ & JX442035 & JX442039 & - \\
\hline Bambusicola massarinia & MFLUCC 11-0389 ${ }^{\mathrm{T}}$ & JX442037 & JX442041 & - \\
\hline Bambusicola splendida & MFLUCC 11-0439 ${ }^{T}$ & JX442038 & JX442042 & - \\
\hline Bambusistroma didymosporum & MFLUCC 13-0862 ${ }^{\mathrm{T}}$ & KP761730 & KP761737 & KP761721 \\
\hline Botryosphaeria dothidea & CBS $115476^{T}$ & NG_027577 & DQ677998 & DQ677944 \\
\hline Capnodium salicinum & CBS $131.34^{\mathrm{T}}$ & DQ6778050 & DQ677997 & - \\
\hline Clypeoloculus akitaensis & CBS $139681^{T}$ & $\mathrm{AB} 807543$ & $\mathrm{AB} 797253$ & - \\
\hline Cucurbitaria berberidis & CBS 363.93 & GQ387606 & GQ387545 & - \\
\hline Cucurbitaria berberidis & CBS 394.84 & GQ387605 & GQ387544 & - \\
\hline Cystocoleus ebeneus & L161 & EU048578 & EU048571 & - \\
\hline Delitschia didyma & UME 31411 & DQ384090 & NG_016519 & - \\
\hline
\end{tabular}




\begin{tabular}{|c|c|c|c|c|}
\hline \multirow{2}{*}{$\operatorname{Taxon}^{1}$} & \multirow{2}{*}{ Strain number } & \multicolumn{3}{|c|}{ GenBank accession numbers } \\
\hline & & LSU & SSU & RPB2 \\
\hline Delitschia winteri & CBS 225.62 ${ }^{\mathrm{T}}$ & DQ678077 & DQ678026 & DQ677975 \\
\hline Deniquelata barringtoniae & MFLUCC 11-0422 ${ }^{\mathrm{T}}$ & NG_042696 & JX254656 & - \\
\hline Dictyosporium alatum & ATCC $34953^{\mathrm{T}}$ & DQ018101 & DQ018080 & - \\
\hline Dictyosporium elegans & NBRC 32502 & DQ018100 & DQ018079 & - \\
\hline Dictyosporium strelitziae & CBS $123359^{T}$ & FJ839653 & - & - \\
\hline Didymella exigua & CBS $183.55^{\mathrm{T}}$ & EU754155 & EU754056 & - \\
\hline Didymosphaeria rubi-ulmifolii & MFLUCC 14-0024 T & KJ436585 & KJ436587 & - \\
\hline Digitodesmium bambusicola & CBS $1102799^{\mathrm{T}}$ & DQ018103 & - & - \\
\hline Erythrodecton granulatum & Ertz 9908 (BR) & EU704090 & - & EU704022 \\
\hline Extremus antarcticus & CCFEE5312 & KF310020 & - & KF310086 \\
\hline Falciformispora lignatilis & BCC 21117 & GU371826 & GU371834 & - \\
\hline Falciformispora senegalensis & CBS 196.79 ${ }^{\mathrm{T}}$ & KF015631 & KF015636 & KF015717 \\
\hline Fissuroma maculans & MFLUCC 10-0886 & NG_042598 & JN846738 & - \\
\hline Fuscostagonospora sasae & CBS $139687^{\mathrm{T}}$ & AB807548 & AB797258 & - \\
\hline Halomassarina thalassiae & JK 5262D & GU301816 & - & - \\
\hline Halotthia posidoniae & BBH 22481 & GU479786 & GU479752 & - \\
\hline Helicascus elaterascuss & HKUCC 7769 & AY787934 & AF053727 & - \\
\hline Helicascus kanaloanus & A237 & - & AF053729 & - \\
\hline Hysterium angustatum & CBS 236.34 ${ }^{\mathrm{T}}$ & FJ161180 & GU397359 & FJ161117 \\
\hline Inflatispora pseudostromatica & CBS $123110{ }^{T}$ & JN231131 & JN231132 & JN231133 \\
\hline Jahnula seychellensis & SS2113 & EF175665 & EF175643 & - \\
\hline Jalapriya toruloides & CBS 209.65 & DQ018104 & DQ018081 & - \\
\hline Keissleriella cladophila & CBS $104.55^{\mathrm{T}}$ & GU301822 & GU296155 & GU371735 \\
\hline Latorua caligans & CBS 576.65 ${ }^{\mathrm{T}}$ & KR873266 & - & - \\
\hline Latorua grootfonteinensis & CBS 369.72 ${ }^{\mathrm{T}}$ & KR873267 & - & - \\
\hline Lentithecium fluviatile & CBS 122367 & GU301825 & GU296158 & - \\
\hline Lentithecium lineare & IFRD 2008 & FJ795435 & FJ795478 & - \\
\hline Leptosphaeria doliolum & CBS 505.75 ${ }^{\mathrm{T}}$ & GQ387576 & GQ387515 & KT389640 \\
\hline Leptoxyphium cacuminum & MFLUCC 10-0049 ${ }^{\mathrm{T}}$ & JN832602 & JN832587 & - \\
\hline Lindgomyces ingoldianus & ATCC $200398^{T}$ & AB521736 & AB521719 & - \\
\hline Longipedicellata aptrootii & MFLUCC 16-0384 & KY066738 & KY066740 & - \\
\hline Longipedicellata aptrootii & MFLUCC 16-0244 & KY066739 & KY066741 & KY066737 \\
\hline Longipedicellata aptrootii & MFLUCC 10-0297 ${ }^{\mathrm{T}}$ & KU238894 & KU238895 & KU238891 \\
\hline Lophiostoma macrostomum & JCM 13544 & AB619010 & AB618691 & JN993491 \\
\hline Lophiotrema lignicola & CBS $122364^{T}$ & FJ795445 & GU296166 & FJ795462 \\
\hline Lophiotrema nucula & CBS $627.86^{T}$ & GU301837 & - & GU371792 \\
\hline Lophium mytilinum & AFTOL-ID $1609^{T}$ & DQ678081 & DQ678030 & DQ677979 \\
\hline Macrodiplodiopsis desmazieri & CBS 221.37 & JX681100 & - & - \\
\hline Macrodiplodiopsis desmazieri & CBS $140062^{T}$ & KR873272 & - & - \\
\hline Magnicamarosporium iriomotense & KT $2822^{T}$ & AB807509 & AB797219 & - \\
\hline Massaria gigantispora & CBS 125593 & HQ599397 & HQ599447 & - \\
\hline Massaria inquinans & CBS 125591 ET & HQ599400 & HQ599442 & - \\
\hline Massarina eburnea & CBS 473.64 & GU301840 & GU296170 & GU371732 \\
\hline Matsushimamyces bohaniensis & CBEC 001 ${ }^{\mathrm{T}}$ & KR350633 & - & - \\
\hline Matsushimamyces venustum & CBS 140212 & KT428158 & - & - \\
\hline Mauritiana rhizophorae & BCC 28866 & GU371824 & GU371832 & - \\
\hline Melanomma pulvis-pyrius & CBS 371.75 & GU301845 & FJ201989 & GU371798 \\
\hline Monodictys capensis & CBS 134928 & AB807551 & AB797261 & - \\
\hline Montagnula aloes & CPC $19671^{T}$ & JX069847 & - & - \\
\hline Morosphaeria ramunculicola & BCC 18404 & GQ925853 & GQ925838 & - \\
\hline Morosphaeria velatispora & BCC $17059^{T}$ & GQ925852 & GQ925841 & - \\
\hline Multilocularia bambusae & MFLUCC 11-0180 ${ }^{\mathrm{T}}$ & KU693438 & KU693442 & - \\
\hline Multiseptospora thailandica & MFLUCC 11-0183 ${ }^{\mathrm{T}}$ & KP744490 & KP753955 & - \\
\hline Murilentithecium clematidis & MFLUCC 14-0561 ${ }^{\mathrm{T}}$ & KM408758 & KM408760 & KM454446 \\
\hline Myriangium duriaei & CBS 260.36 & NG_027579 & AF242266 & KT216528 \\
\hline Myriangium hisparanicum & CPC 18561 & JN940391 & JN940562 & - \\
\hline Mytilinidion rhenanum & CBS $135.34^{T}$ & FJ161175 & FJ161136 & FJ161115 \\
\hline Neoastrosphaeriella krabiensis & MFLUCC 11-0025 ${ }^{\mathrm{T}}$ & JN846729 & JN846739 & - \\
\hline Neobambusicola strelitziae & CBS $138869^{\mathrm{T}}$ & KP004495 & - & - \\
\hline Neokalmusia brevispora & CBS 120248 & AB524600 & AB524459 & AB539099 \\
\hline
\end{tabular}




\begin{tabular}{|c|c|c|c|c|}
\hline \multirow{2}{*}{$\operatorname{Taxon}^{1}$} & \multirow{2}{*}{ Strain number } & \multicolumn{3}{|c|}{ GenBank accession numbers } \\
\hline & & LSU & SSU & RPB2 \\
\hline Neomassariosphaeria typhicola & CBS 609.86 ${ }^{\mathrm{T}}$ & EF165033 & EF165037 & EF165041 \\
\hline Neottiosporina paspali & CBS 331.37 & EU754172 & EU754073 & GU371779 \\
\hline Parabambusicola bambusina & KT 2637 & AB807538 & AB797248 & - \\
\hline Parameliola acaciae & MFLU 15-0378 & KU285142 & - & - \\
\hline Parameliola dimocarpi & MFLU 15-0045 & KU285142 & - & - \\
\hline Periconia homothallica & CBS $139698^{\mathrm{T}}$ & AB807565 & AB797275 & - \\
\hline Periconia pseudodigitata & CBS $139699^{\mathrm{T}}$ & AB807564 & AB797274 & - \\
\hline Phaeodimeriella cissampeli & MFLU 16-0558 & KU746806 & KU746808 & KU746810 \\
\hline Phaeodimeriella dilleniae & MFLU 14-0013 & KU746805 & KU746807 & KU746809 \\
\hline Phyllosticta ampelicida & CBS $1116455^{\mathrm{T}}$ & DQ377876 & EU673223 & - \\
\hline Piedraia hortae & CBS 480.64 ${ }^{\mathrm{T}}$ & GU214466 & AY016349 & KF902289 \\
\hline Pleomassaria siparia & AFTOL-ID $1600^{T}$ & DQ678078 & DQ678027 & DQ677976 \\
\hline Plespora herbarum & CBS $191.86^{T}$ & GU238160 & GU238232 & DQ247794 \\
\hline Polyschema congolensis & CBS 542.73 ${ }^{\mathrm{T}}$ & EF204502 & - & EF204486 \\
\hline Polyschema larviformis & CBS 463.88 & EF204503 & - & - \\
\hline Polyschema sclerotigenum & UTHSC:DI14-305 & KP769976 & - & - \\
\hline Polyschema terricola & CBS 301.65 ${ }^{\mathrm{T}}$ & EF204504 & EF204519 & EF204487 \\
\hline Preussia funiculata & CBS 659.74 ${ }^{\mathrm{T}}$ & GU301864 & GU296187 & GU371799 \\
\hline Pseudoasteromassaria fagi & MAFF $245222^{T}$ & LC061589 & LC061584 & - \\
\hline Pseudoasteromassaria fagi & MAFF 245221 & LC061590 & LC061585 & - \\
\hline Pseudocoleophoma calamagrostidis & CBS $139700^{\mathrm{T}}$ & LC014609 & LC014604 & - \\
\hline Pseudocoleophoma polygonicola & CBS $139701^{\mathrm{T}}$ & AB807546 & AB797256 & - \\
\hline Pseudodictyosporium elegans & CBS 688.93 ${ }^{\mathrm{T}}$ & DQ018106 & DQ018084 & - \\
\hline Pseudodictyosporium wauense & NBRC 30078 & DQ018105 & DQ018083 & - \\
\hline Pseudomassariosphaeria grandispora & CBS 613.86 & EF165034 & GU296172 & GU371725 \\
\hline Pseudomonodictys tectonae & MFLUCC 12-0552 ${ }^{\mathrm{T}}$ & KT285573 & KT285574 & KT285572 \\
\hline Pseudostrickeria muriformis & MFLUCC 13-0764 T & KT934254 & KT934258 & - \\
\hline Pseudoxylomyces elegans & MAFF $243852^{T}$ & AB807598 & AB797308 & - \\
\hline Psiloglonium clavisporum & CBS 306.38 ${ }^{\mathrm{T}}$ & FJ469672 & GU296191 & - \\
\hline Ramularia endophylla & CBS $113265^{\mathrm{T}}$ & KF251833 & - & KF252332 \\
\hline Rasutoria pseudotsugae & rapssd & EF114704 & EF114729 & - \\
\hline Rasutoria tsugae & ratstk & EF114705 & EF114730 & GU371809 \\
\hline Rimora mangrovei & JK 5246A & GU301868 & GU296193 & GU371759 \\
\hline Roccella fuciformis & Tehler 8171 & FJ638979 & - & FJ639038 \\
\hline Schismatomma decolorans & Ertz 5003 (BR) & NG_027622 & NG_013155 & - \\
\hline Schizothyrium pomi & CBS 406.61 & EF134949 & - & - \\
\hline Sigarispora arundinis & CBS $621.86^{\mathrm{T}}$ & DQ782384 & DQ782383 & DQ782386 \\
\hline Stagonospora pseudocaricis & CBS $135132^{\mathrm{T}}$ & KF251762 & - & KF252264 \\
\hline Sulcatispora acerina & KT $2982^{T}$ & LC014610 & LC014605 & - \\
\hline Sulcatispora berchemiae & KT $1607^{T}$ & AB807534 & AB797244 & - \\
\hline Teratosphaeria fibrillosa & CBS 121707 & GU323213 & GU296199 & - \\
\hline Tetraploa aristata & CBS 996.70 & AB524627 & AB524486 & - \\
\hline Tetraplosphaeria sasicola & $\mathrm{JCM} 13167^{\mathrm{T}}$ & AB524631 & AB524490 & - \\
\hline Trematosphaeria grisea & CBS 332.50 ${ }^{\mathrm{T}}$ & KF015614 & KF015641 & KF015720 \\
\hline Trematosphaeria pertusa & CBS $122368^{\mathrm{T}}$ & FJ201990 & FJ201991 & FJ795476 \\
\hline Tubeufia chiangmaiensis & MFLUCC 11-0514 ${ }^{\mathrm{T}}$ & KF301538 & KF301543 & - \\
\hline Tubeufia javanica & MFLUCC 12-0545 ${ }^{\mathrm{T}}$ & KJ880036 & KJ880035 & - \\
\hline Uwebraunia commune & CBS 114238 & EU019267 & GU214526 & - \\
\hline
\end{tabular}

1 The ex-type strains are in bold, the new family introduced in this study is indicated in red.

\section{Results}

\section{Topology of phylogenetic analysis}

The final alignment of the combined dataset consisted of 137 strains and 3002 characters (LSU 903 characters, SSU 1033 characters, and RPB2 1066 characters). The tree topology for LSU was compared visually to confirm that it is correlated with the overall tree topology obtained from the combined alignment at the family level. The topology at family levels is similar to previous studies (Sharma et al. 2015, Boonmee et al. 2016, Li et al. 2016). The Bayesian analysis and maximum likelihood topology are comparable, except for Fuscostagonospora sasae (CBS 139687) 


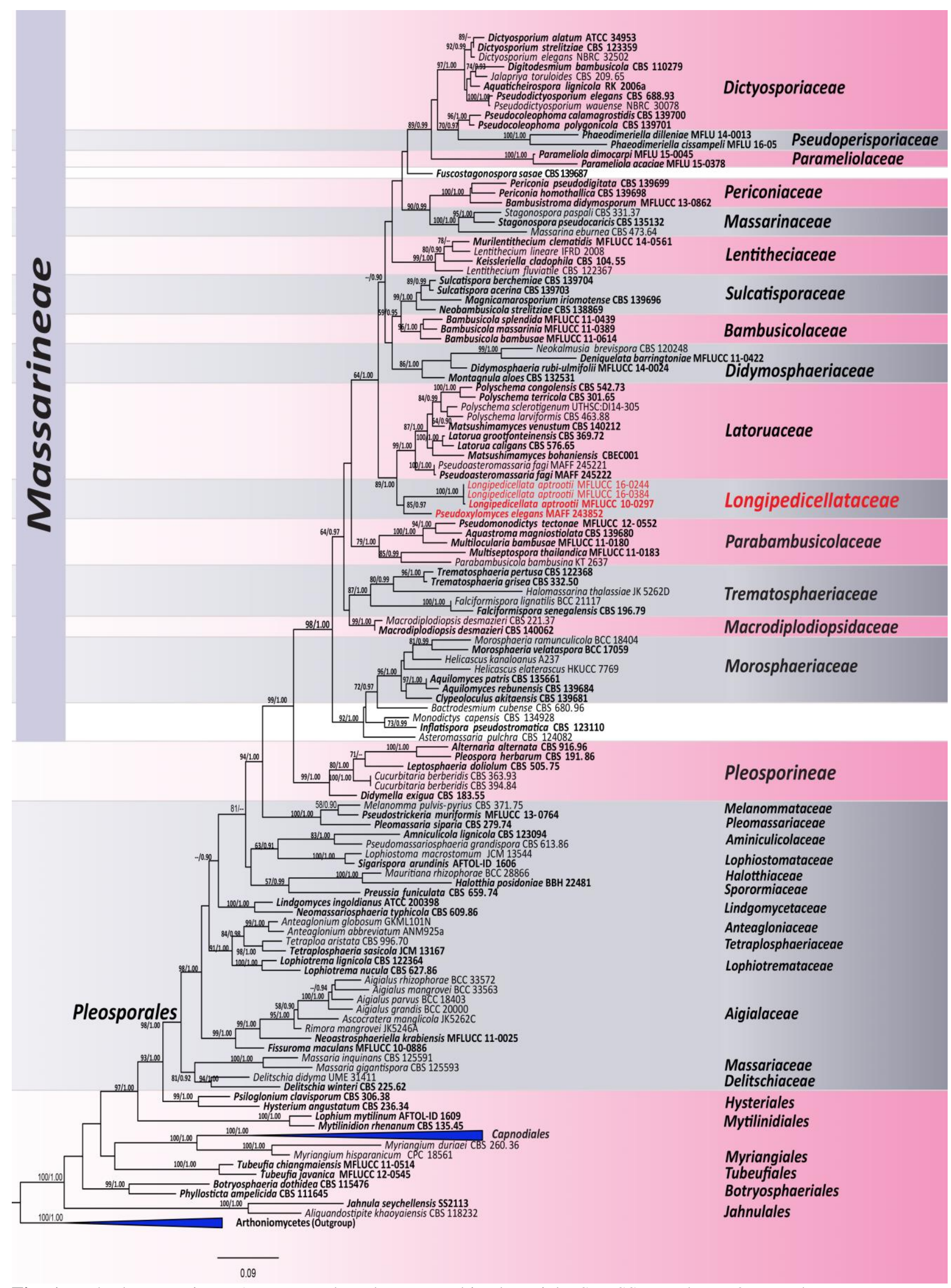

Fig. 1 - The best scoring RAxML tree based on a combined partial LSU, SSU, and RPB2 gene datasets. Bootstrap values $\geq 70 \%$ from the maximum likelihood (ML) analysis are followed by Bayesian posterior probabilities (PP) values $\geq 0.90$. The tree is rooted with Arthoniomycetes as the outgroup taxa. The family Longipedicellataceae which determined in this study is indicated in red. The ex-type and references strains are indicated in black bold. Hyphen (-) represents support values $\leq 70 \% / 0.90$. 
being a sister taxon to Periconiaceae and Massarinaceae in the Bayesian analysis (data not shown). The best scoring RAxML tree had a final likelihood value of -49637.912100 and is shown in Fig. 1.

Our analysis included 109 strains from Pleosporales, which forms a sister group to Hysteriales with high support (93\% ML/1.00 PP). Three strains of Longipedicellata aptrootii form a sister group to Pseudoxylomyces elegans (MAFF 243852) with strong support (85\% ML/0.97 PP) and we introduce the family Longipedicellataceae for these two genera. Longipedicellataceae clusters in the suborder Massarineae (Pleosporales). Longipedicellataceae further clusters with Latoruaceae with high support (89\% ML/1.00 PP).

Furthermore, in our analysis Phaeodimeriella (Pseudoperisporiaceae) (Mapook et al. 2016) and two strains of Parameliola (Li et al. 2016) form a distinct lineage, sister to Dictyosporiaceae. Phaeodimeriella dilleniae and Ph. cissampeli form a sister clade with Pseudocolephoma species (70\% ML/ 0.97 PP) which Tanaka et al. (2015) placed in Dictyosporiaceae. Parameliola formed a clade with Phaeodimeriella and Pseudocolephoma, however there is not good statistic support for the internal clade. Thus, our results provide evidence for the use of Pseudoperisporiaceae and Parameliolaceae. Pseudocolephoma may need transferring to Pseudoperisporiaceae following further studies. Fuscostagonospora sasae may be a new family, but we refrain from introducing it here as there is only one representative strain.

\section{Divergence time estimates}

The topologies from the BEAST analysis are congruent with the result from the maximum likelihood analysis (Fig. 1). The divergence time estimates based on scenarios 2 (one secondary and two fossil calibrations) are shown in Fig. 2, the calibration points are indicated with red dots. The horizontal bars at the nodes represents the $95 \%$ highest posterior probability (HPD) intervals for the age of that node. The mean dates estimated in this study mostly rely on highest posterior density of previous studies (Beimforde et al. 2014, Pérez-Ortega et al. 2016). The divergence times

Table 2 Divergence time estimates of Dothideomycetes lineages obtained from BEAST analysis using calibrations from amber (Aigialus sp., Metacapnodiaceae). For each divergence, the median and the 95\% Highest Posterior Density are provided. Divergence times are provided in millions of years (Mya). The geological time scales are given. The node numbers correspond to numbers used in Fig. 2 to show placement in the chronogram.

\begin{tabular}{|c|c|c|c|c|c|c|c|c|c|}
\hline \multirow[t]{2}{*}{ Nodes } & & \multicolumn{4}{|c|}{ This study } & \multirow{2}{*}{$\begin{array}{c}\text { Gueidan } \\
\text { et al. } \\
(2011)\end{array}$} & \multirow{2}{*}{$\begin{array}{l}\text { Prieto } \\
\& \\
\text { Wedin } \\
(2013) \\
\end{array}$} & \multirow{2}{*}{$\begin{array}{l}\text { Beimforde } \\
\text { et al. } \\
(2014) \\
\text { (Cali 2) }\end{array}$} & \multirow{2}{*}{$\begin{array}{c}\text { Pérez- } \\
\text { Ortega } \\
\text { et al. } \\
(\mathbf{2 0 1 6})\end{array}$} \\
\hline & & (1 fossil) & $\begin{array}{l}\text { Georogical } \\
\text { time scales }\end{array}$ & $\begin{array}{c}2 \\
(2 \text { fossil }) \\
\end{array}$ & $\begin{array}{l}\text { Georogical } \\
\text { time scales }\end{array}$ & & & & \\
\hline 1 & $\begin{array}{l}\text { Dothideomycetes- } \\
\text { Arthoniomycetes }\end{array}$ & $\begin{array}{c}270 \\
(167-369)\end{array}$ & Permian & $\begin{array}{c}317 \\
(236-397)\end{array}$ & $\begin{array}{l}\text { Carboni- } \\
\text { ferous }\end{array}$ & 362 & 302 & 362 & 313 \\
\hline 2 & $\begin{array}{l}\text { Capnodiales crown } \\
\text { group }\end{array}$ & $\begin{array}{c}122 \\
(65-181)\end{array}$ & $\begin{array}{c}\text { Middle } \\
\text { Cretaceous }\end{array}$ & $\begin{array}{c}147 \\
(102-202)\end{array}$ & $\begin{array}{c}\text { Late } \\
\text { Jurassic }\end{array}$ & - & - & $\sim 160$ & - \\
\hline 3 & $\begin{array}{l}\text { Aigialaceae - } \\
\text { Aigialus sp. }\end{array}$ & $\begin{array}{c}22 \\
(10-39) \\
\end{array}$ & Miocene & $\begin{array}{c}39 \\
(35-49) \\
\end{array}$ & Eocene & - & - & - & - \\
\hline 4 & $\begin{array}{l}\text { Dothideomycetes } \\
\text { crown group }\end{array}$ & $\begin{array}{c}244 \\
(148-347)\end{array}$ & $\begin{array}{l}\text { Middle } \\
\text { Triassic }\end{array}$ & $\begin{array}{c}293 \\
(213-371)\end{array}$ & Permian & 338 & 174 & 350 & 290 \\
\hline 5 & $\begin{array}{l}\text { Pleosporales crown } \\
\text { group }\end{array}$ & $\begin{array}{c}170 \\
(95-247)\end{array}$ & $\begin{array}{l}\text { Middle } \\
\text { Jurassic }\end{array}$ & $\begin{array}{c}211 \\
(153-277)\end{array}$ & $\begin{array}{c}\text { Late } \\
\text { Triassic }\end{array}$ & $\sim 190$ & - & $\sim 150$ & - \\
\hline 6 & $\begin{array}{l}\text { Massarineae- } \\
\text { Plesporineae }\end{array}$ & $\begin{array}{c}115 \\
(65-168)\end{array}$ & Cretaceous & $\begin{array}{c}144 \\
(104-191)\end{array}$ & $\begin{array}{c}\text { Early } \\
\text { Cretaceous }\end{array}$ & - & - & - & - \\
\hline 7 & $\begin{array}{l}\text { Massarineae crown } \\
\text { group }\end{array}$ & $\begin{array}{c}104 \\
(57-151)\end{array}$ & Cretaceous & $\begin{array}{c}130 \\
(96-174)\end{array}$ & Cretaceous & - & - & - & - \\
\hline 8 & $\begin{array}{l}\text { Latoruaceae- } \\
\text { Longipedicellataceae }\end{array}$ & $\begin{array}{c}59 \\
(30-94) \\
\end{array}$ & Paleocene & $\begin{array}{c}75 \\
(48-107) \\
\end{array}$ & Cretaceous & - & - & - & - \\
\hline 9 & $\begin{array}{l}\text { Longipedicellataceae } \\
\text { crown group }\end{array}$ & $\begin{array}{c}44 \\
(15-77) \\
\end{array}$ & Eocene & $\begin{array}{c}56 \\
(25-89) \\
\end{array}$ & Eocene & - & - & - & - \\
\hline 10 & $\begin{array}{l}\text { Latoruaceae crown } \\
\text { group }\end{array}$ & $\begin{array}{c}42 \\
(22-70)\end{array}$ & Eocene & $\begin{array}{c}53 \\
(31-78) \\
\end{array}$ & Eocene & - & - & - & - \\
\hline
\end{tabular}




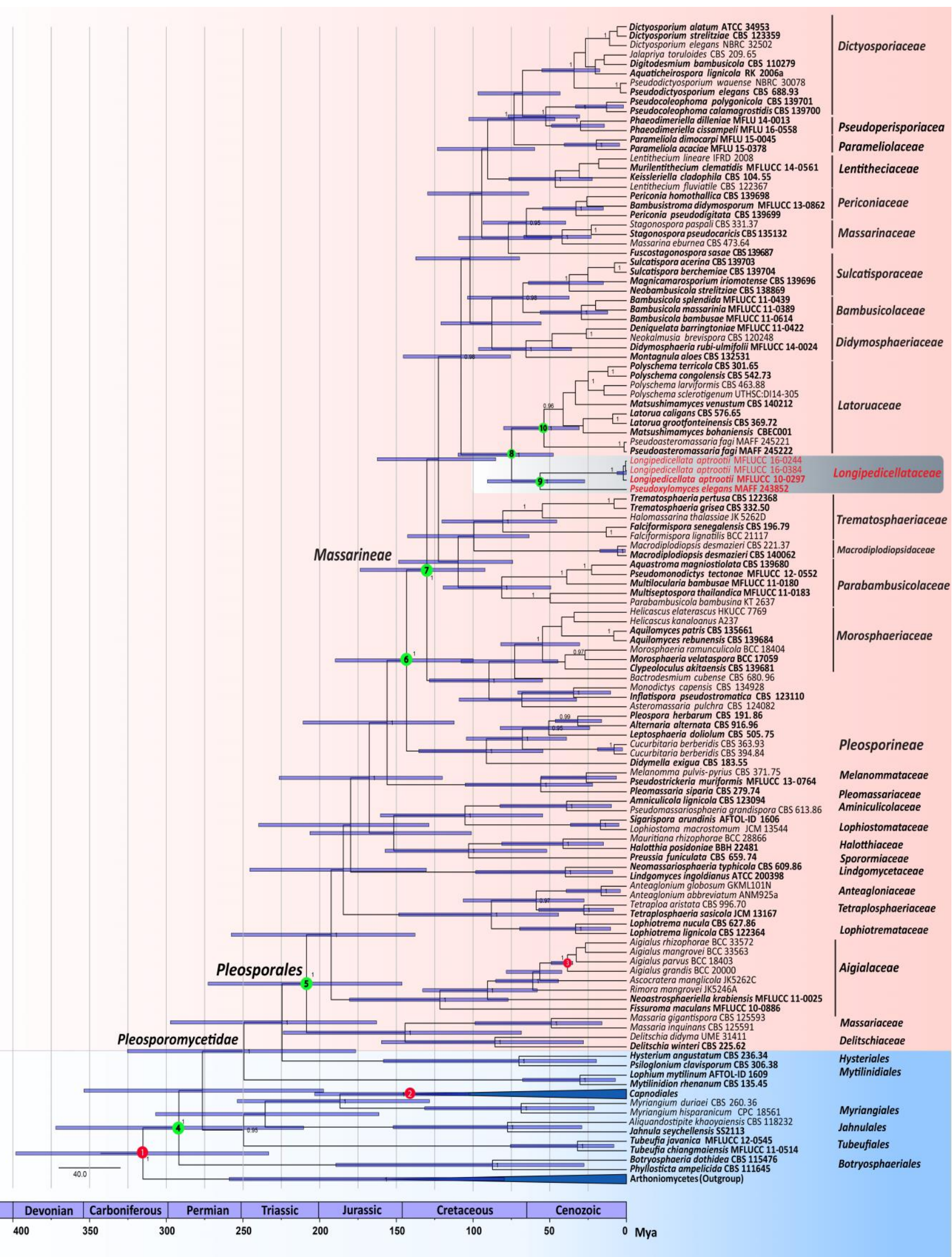

Fig. 2 - Maximum clade credibility (MCC) tree with divergence times estimates for main groups of the Dothideomycetes obtained from a Bayesian approach (BEAST) using four fossil calibrations. Numbers at nodes indicate posterior probabilities (PP) for node support. Bars correspond to the $95 \%$ highest posterior density (HPD) intervals. The fossil minimum age constraints and second calibrations used in this study are marked with red dots. Geological time scales are given at the base, together with scale in million years ago (Mya). The nodes specially discussed in this study are marked with green dots. Longipedicellataceae is highlighted in gray. For estimated median ages of numbered nodes, see Table 2 . 
of the dating analysis are listed in Table 2 . The crown group discussed below refers to all the descendants of the last common ancestor living in the group. The maximum clade creditability tree indicates that the split of Dothideomycetes and Arthoniomycetes occurred around 317 (236-397) Mya, in the Carboniferous. The split between Massarineae and Pleosporineae, two major suborders within Pleosporales occurred approximately 144 (104-191) Mya, in the Cretaceous. The Pleosporales crown group evolved around 211 (153-277) Mya during the Triassic. In this study, we focus on the suborder Massarineae which evolved around 130 (96-174) Mya, during the Cretaceous. Based on our analysis, the families within Massarineae mostly evolved in the Cenozoic era (present around 66 Mya), with the exception of Trematosphaeriaceae, which evolved in the late Cretaceous (80 Mya). Latoruaceae and Longipedicellataceae diverged around 75 (48-107) Mya in the late Cretaceous period. The common ancestor of living representative of Longipedicellataceae strains collected from freshwater (crown group) occurred around 56 (25-89) Mya, in the Cenozoic. Latoruaceae, is a related family, known from soil and hot springs with a crown age around 53 (31-78) Mya, in the Eocene.

\section{Taxonomy}

Longipedicellataceae Phukhams., J. Bhat \& K.D. Hyde, fam. nov.

Index Fungorum Number: IF552532, Facesoffungi number: FoF 01408

Type genus - Longipedicellata Zhang, K.D. Hyde \& J.K. Liu Phytotaxa 247 (2): 104 (2016)

Saprobic on dead and submerged woody material. Sexual morph Ascomata semi-immersed to erumpent in host tissue, coriaceous, solitary, scattered, sometimes embedded under a pseudoclypeus, subglobose to ellipsoidal, black to brown, ostiolate. Peridium multi-layered, of black to brown cells of textura angularis, sometimes textura prismatica, somewhat carbonaceous, thin, easy to break. Hamathecium comprising few, long, broad, septate, branched, cellular pseudoparaphyses, surrouding asci and along the inner layer of peridium. Asci 8-spored, bitunicate, fisitunicate, clavate, long-pedicellate, bulbous, thin-walled, with an apical ocular chamber. Ascospores bi-seriate, overlapping, ellipsoidal, narrowly subfusiform, conical at apex, hyaline, 1septate, constricted at septum, guttulate, smooth-walled. Asexual morph: Hyphomycetous Colonies black to dark-brown, circular, effuse. Mycelium composed of smooth, hyaline to dark brown, septate, branched, hyphae, swollen in ovoid cells. Chlamydospores acropetal, catenate, doliiform, branched, subglubose to oval, rough-walled, in long chains, brown to reddish-brown.

Notes - Longipedicellataceae includes the genera Longipedicellata and Pseudoxylomyces which are reported from aquatic habitats, as saprobes on woody substrates. Longipedicellataceae is characterized by semi-immersed or erumpent, clypeate ascomata on the host tissues, with black to brown ostiolates, clavate asci with long pedicels, and 2-celled, hyaline ascospores. Chlamydospore formation in Longipedicellata and Pseudoxylomyces is also significant. All the strains formed a well-supported clade (89\%ML/1.00PP) basal to Latoruaceae within the suborder Massarineae (Pleosporales). Longipedicellata and Pseudoxylomyces clustered together with good support (85\% ML/0.97 PP).

Longipedicellata Zhang, K.D. Hyde \& J.K. Liu Phytotaxa 247 (2): 104 (2016)

Index Fungorum Numbers: IF551685, Facesoffungi number: FoF 02665

Type species - Longipedicellata aptrootii (Hyde \& Wong) Zhang, K.D. Hyde \& J.K. Liu

Saprobic on dead and submerged woody material in freshwater. Sexual morph: Ascomata lenticular, immersed, beneath a blackened pseudoclypeus, scattered or clustered, coriaceous, black to brown, sometimes ostiolate. Peridium comprising multi-layered, black to brown cells of textura angularis, outer layer somewhat carbonaceous, inner layer composed of subhyaline gelatinous cells, easy to break. Hamathecium comprising few, long, broad, transversely septate, branched, cellular pseudoparaphyses, surrounding asci and along the inner layer of peridium. Asci 8-spored, bitunicate, fisitunicate, clavate, long-pedicellate, thin-walled, with an ocular chamber. Ascospores 8-spored, bi-seriate, overlapping, ellipsoid, narrowly subfusiform, conical at apex, hyaline, with 1transverse-septa, constricted at the septum, guttulate at the centre of each cell, surrounded by 
mucilaginous sheath (adaptation from Zhang et al. 2016). Asexual morph: Hyphomycetous. Colonies dark-brown to black, circular, effuse, with serrate margin. Mycelium composed of smooth, hyaline to dark brown, septate, branched hyphae, flexuous, slightly filiform on agar suface, septate, branched, thick-walled, smooth, pale brown, septate, integrated, terminal or sometimes intercalary, gangliar-type (Bhat 2010), smooth to verrucose, hyaline to pale brown, tranformed into chlamydospores. Conidia chlamydosporous monilioid, in acropetal chains, doliiform, subglubose to oval, rough-walled, brown to reddish-brown.

Longipedicellata aptrootii (Hyde \& Wong) Zhang, K.D. Hyde \& J.K. Liu, Phytotaxa 247(2): 104 (2016)

Index Fungorum number: IF551686 Facesoffungi number: FoF: 01273

Fig. 3

三Didymella aptrootii Hyde \& S.W. Wong, Australas. Mycol. 18(3): 54 (1999)

Holotype - IFRD (HKU(M) 3333)!)

Saprobic on dead and submerged stem of Bambusodeae. Sexual morph: Ascomata 115-143 $\mu \mathrm{m}$ high $\times 182-269 \mu \mathrm{m}$ diam. $(\bar{x}=125 \times 235 \mu \mathrm{m}, \mathrm{n}=10)$, semi-immersed to erumpent in the host tissue, coriaceous, solitary, scattered or in small groups, sometimes under the clypeus, subglobose to ellipsoidal, rough-walled, black to brown, sometimes ostiolate. Peridium 7-15(-25) $\mu \mathrm{m}$ wide, composed of 2-4 layers of black to brown cells of textura angularis and textura prismatica, outer layer somewhat carbonaceous, inner layer composed of subhyaline gelatinous cells, up to $10 \mu \mathrm{m}$, thin, easy to break. Hamathecium comprising few, 1.1-2.8 $\mu \mathrm{m}$ wide $(\bar{x}=2 \mu \mathrm{m}, \mathrm{n}=20)$, long, broad, septate, branched, cellular pseudoparaphyses, surrounding asci and along the inner layer of peridium. Asci 63-170 × 14-25 $\mu \mathrm{m}(\bar{x}=81 \times 18 \mu \mathrm{m}, \mathrm{n}=70), 8$-spored, bitunicate, fisitunicate, clavate, long-pedicellate, $28-38 \mu \mathrm{m}$, bulbous, thin-walled, shallow ocular chamber up to $1.8 \mu \mathrm{m}$ wide $\times 2 \mu \mathrm{m}$ high. Ascospores 19-26 $\mu \mathrm{m} \times 7-11 \mu \mathrm{m}(\bar{x}=23 \times 9 \mu \mathrm{m}, \mathrm{n}=70)$, overlapping biseriate, ellipsoid, narrowly subfusiform, conical at the apex, hyaline, 1-septate, constricted at the septum, guttulate in the centre of cells, smooth-walled. Asexual morph: Hyphomycetous. Mycelium producing chalmydospores and chlamydospore-like structures after two months, smoothwalled, hyaline to dark brown, septate, branched, hyphae, with ovoid cells $0.7-2(-3) \mu \mathrm{m}$ diam.. Colonies black to dark-brown, circular, effuse, flexuous, slightly filiform on agar suface, septate, branched, thick-walled, smooth, pale brown, gangliar-type, integrated, terminal or sometimes intercalary, smooth to verrucose, hyaline to pale brown,.tranformed into chlamydospores. Conidia chlamydosporous 4-14 $\times 5-11 \mu \mathrm{m}$ diam. $(\bar{x}=9 \times 11 \mu \mathrm{m}, \mathrm{n}=80)$, monilioid, in long acropetal chains, catenate, doliiform, branched, subglubose to oval, rough-walled, brown to reddish-brown.

Culture characteristics - Ascospores germinating on PDA within $24 \mathrm{~h}$, with germ tubes developing from apical and basal cells. Colonies on PDA reaching $20 \mathrm{~mm}$ in diameter after four weeks at $25^{\circ} \mathrm{C}$. Colonies black, with arial white mycelium, umbonate at the centre, with circular, friable, dark brown margin; reverse dark brown to black, with a horizontal, deep slit in the agar. Chlamydospore-like structures formed in culture after two months of incubation.

Material examined - Hong Kong, New Territories, Plover Cove Reservoir, on submerged bamboo, 15 November 1996, K.D. Hyde (IFRD (HKU(M) 3333)!); Thailand, Chiang Rai, Hui Kang Pla Waterfall, on submerged bamboo (Poaceae), 18 January 2010, Huang Zhang (MFLU 100162!, reference specimen), ex-culture: MFLUCC 10-0297; ibid (MFLU 10-0163!, MFLU 100164!, MFLU 16-0032!); Chiang Rai Province, on dead and submerged stem of Bambusodeae (Poaceae), 14 June 2015, C. Phukhamsakda, CP015 (MFLU 16-0032, paratype), ibid., 15 January 2016, ex-paratype living culture, MFLUCC 16-0384, KMUCC 15-0552.

Notes - The genus Longipedicellata was introduced by Zhang et al. (2016) based on a collection of Didymella aptrootii K.D. Hyde \& S.W. Wong, from a freshwater stream in Thailand. In their phylogenetic analyses, the genus showed a close relationship to Bambusicolaceae with moderate support (65\% ML/0.99 PP). Therefore, Zhang et al. (2016) transferred Didymella aptrootii to Longipedicellata aptrootii and placed it in Bambusicolaceae. We also collected fresh material of Longipedicellata aptrootii. Our collection (MFLUCC 16-0384) was morphologically similar with the type material of $L$. aptrootii. In our analyses, the genus formed a close relationship 
with Pseudoxylomyces elegans (Goh et al.) Kaz. Tanaka \& K. Hiray. basal to Latoruaceae (85\% ML/0.97 PP). The L. aptrootii strain (MFLUCC 16-0384) formed chlamydospore-like structures in culture, and produced conidia in chains at the apex which were similar to Pseudoxylomyces elegans. Pseudoxylomyces elegans also produces chlamydospores in chains at the apex of hypha (Goh et al. 1997).

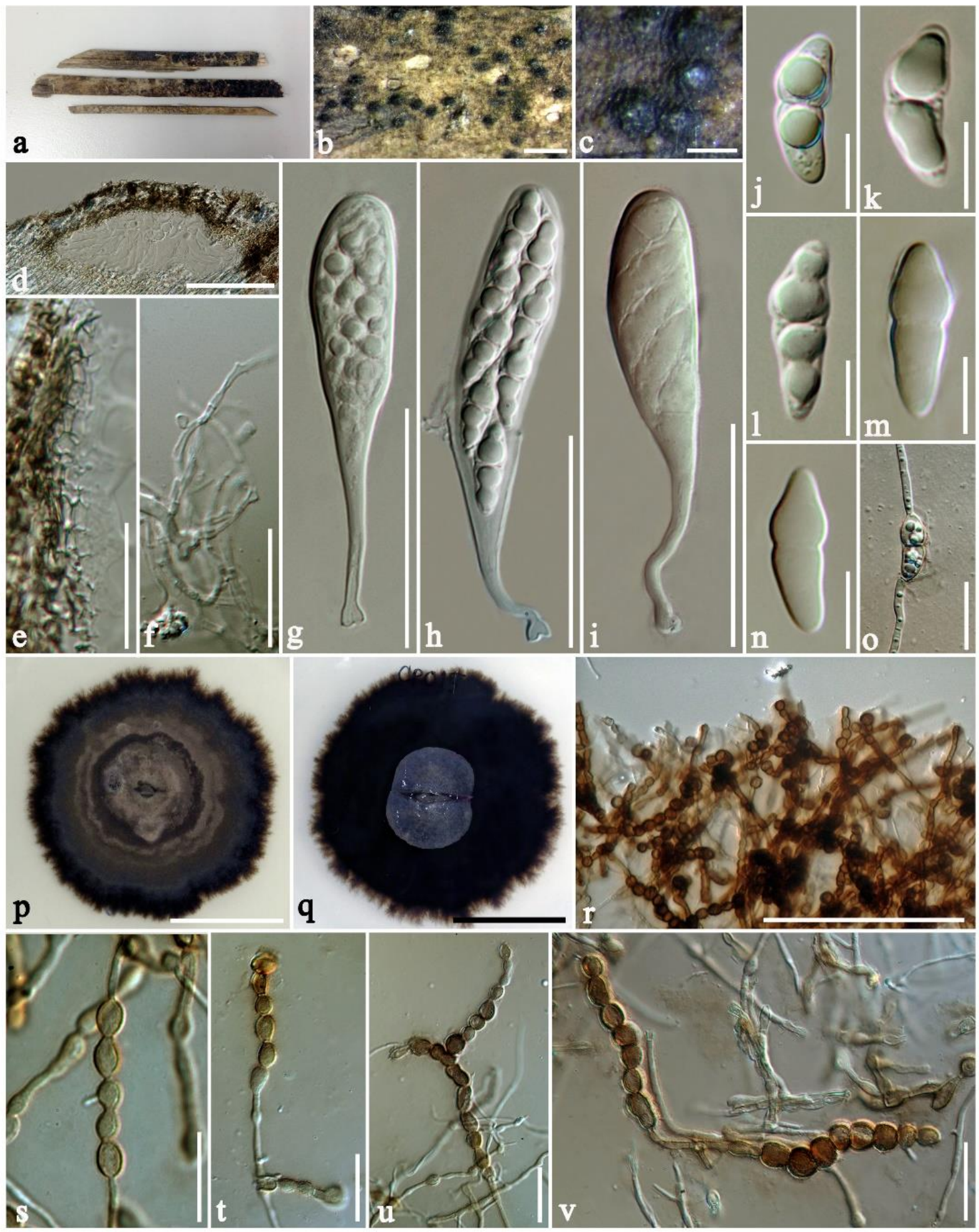

Fig. 3 - Longipedicellata aptrootii (MFLU 16-0032, reference specimen). a Bambusodeae sp. habit. b, c Appearance of ascomata on the host surface. d Vertical section of ascoma. e Section of peridium comprising cells of textura prismatica. f Pseudoparaphyses. $\mathbf{g}-\mathbf{i}$ Asci with long pedicels. $\mathbf{j}-\mathbf{n}$ Development stages of ascospores. o Germinated ascospore. $\mathbf{p}$, $\mathbf{q}$ Culture characters on PDA. $\mathbf{r}$ Close up of culture. $\mathbf{s}$ Chlamydospores. $\mathbf{t}-\mathbf{v}$ Chlamydospore-like conidia in chains. - Bars: $b=500 \mu \mathrm{m}, \mathrm{c}=200 \mu \mathrm{m}, \mathrm{d}=100 \mu \mathrm{m}, \mathrm{e}-\mathrm{f}, \mathrm{o}, \mathrm{s}-\mathrm{v}=20 \mu \mathrm{m}, \mathrm{g}-\mathrm{i}=50 \mu \mathrm{m}, \mathrm{j}-\mathrm{n}=10 \mu \mathrm{m}, \mathrm{p}-\mathrm{q}=20 \mathrm{~cm}$. 
Pseudoxylomyces (Goh, W.H. Ho, K.D. Hyde \& K.M. Tsui) Tanaka \& K. Hirayama., Studies in Mycology 82: 126. 2015.

Type species - Pseudoxylomyces elegans (Goh, W.H. Ho, K.D. Hyde \& K.M. Tsui) Tanaka \& K. Hirayama, Mycol. Res. 101 (11): 1324 (1997)

Holotype - HKU (M) 2777

See Goh et al. 1997 for description, Figs. 15-20.

Notes - Pseudoxylomyces was introduced to accommodate Xylomyces elegans, that clustered in Pleosporales and not Jahnulales. Thus, Xylomyces elegans was synonimyzed as Pseudoxylomyces elegans (Tanaka et al. 2015). Pseudoxylomyces elegans collections are reported from aquatic habitats on decaying wood. Tanaka et al. (2015) showed that Pseudoxylomyces elegans formed a basal clade to Trematosphaeriaceae and then placed it genus incertae sedis in the suborder Massarineae (Pleosporales). In our analysis, P. elegans is inferred as closely related to Longipedicellata aptrootii with strong support (85\% ML/0.97 PP). However, no sexual morph is reported for $P$. elegans. When comparing asexual morphs of the genera, they both form chlamydospores-like structures. Pseudoxylomyces elegans, is characterized by dematiaceous mycelia and conidiophores and chlamydospore-like, thick-walled, terminal or intercalary, septate conidia, which are very similar to the chlamydospore structures seen in Longipedicellata aptrootii. They may even be the same genus.

\section{Discussion}

In this paper, we introduce a new family, Longipedicellataceae, in the suborder Massarineae (Pleosporales). Longipedicellataceae includes the genera Longipedicellata (L. aptrootii) and Pseudoxylomyces (P. elegans) which are found on woody debris in freshwater (Hyde \& Wong 1999, Zhang et al. 2016).

\section{Divergence time estimates}

Pleosporales is the largest order in Dothideomycetes, and species have a highly diverse range of habitat and lifestyles (Zhang et al. 2012, Hyde et al. 2013). Pleosporales is included in the subclass Pleosporomycetidae, and shares a common ancestry with Hysteriales and Mytilinidiales (Boehm et al. 2009, Hyde et al. 2013). The order has unique characteristics in its perithecial ascomata, whereas Hysteriales and Mytilinidiales commonly have hysterothecial, carbonaceous and elongate ascomata (Boehm et al. 2009, Mugambi \& Huhndorf 2009, Lumbsch \& Huhndorf 2010).

Divergence time estimates in this study are based on calibration points for Dothideomycetes and previous studies (Beimforde et al. 2014, McLaughlin \& Spatafora 2015, Pérez-Ortega et al. 2016). In addition, the ages of the order Pleosporales and the divergences time of Longipedicellataceae and related families in Pleoporales were estimated in this study.

Schmidt et al. (2010) reported a Curvularia-like taxon in Ethiopian amber from the Cretaceous period. However, similar dematiaceous hyphomycetes with three horizontal septate conidia can be found in various fungal genera (Ellis 1971, Seifert et al. 2011). Consequently, Beimforde et al. (2014) did not use this fossil for calibration of Pleosporales. Mindell et al. (2007) described Margaretbarromyces dictyosporus, a pleosporalean species from Vancouver Island (Canada). The species colonized fossilized woody bark in rocks dated to the Eocene epoch (56-33 Mya, Mindell et al. 2007, Berbee \& Taylor 2010). Based on the information from the fossil, Mindell et al. (2007) compared this fungus to Cucurbitaria (Cucurbitariaceae, Pleosporales). The morphology is also comparable to extant genera in Pleosporales; Margaretbarromyces dictyosporus is similar to the marine fungus Aigialus (Aigialaceae, Pleosporales). This intertidal genus is typically associated with bark or dead wood in mangroves (Suetrong et al. 2009, Jones et al. 2015). The morphology is similar based on the flask-shaped pseudothecial, uniloculate, immersed ascomata, with ostioles filled with periphyses, a peridium of textura intricata, overlapping asci, and dictyospores with lighter or globose, hyaline, end cells (Kohlmeyer \& Schatz 1985, Hyde 1992, Schmit \& Shearer 2003, Suetrong et al. 2009, Jones et al. 2015). 
In our study, we applied two dating scenarios to compare the unity of applying the fossil calibration to the order Pleosporales. Scenario 1 without calibration of Aigialus crown groups and scenario 2 given the estimates constraint of the Aigialus crown group. The fossil ages were used as minimal age constraints. The divergence time estimation resulted in slightly different dates between the two scenarios tested. In general, scenario 2 resulted in older age estimates than scenario 1 . However, the result from scenario 2 are rather similar to previous studies (Table. 2, Vijaykrishna et al. 2006, Schmidt et al. 2010, Gueidan et al. 2011, Prieto \& Wedin 2013, Beimforde et al. 2014, McLaughlin \& Spatafora 2015, Pérez-Ortega et al. 2016). The split of Dothideomycetes and Arthoniomycetes occurred around 270 Mya in scenario 1, versus 317 Mya in scenario 2. The Pleosporales crown group occurred around 170 Mya in the middle Jurassic, versus 211 Mya in the late Triassic. The order Pleosporales split from Hysteriales in the Triassic (227 Mya) in scenario 2 (Fig. 2). The split between suborder Massarineae-Plesporineae is estimated occur around 115 Mya in scenario 1, versus 144 Mya in scenario 2, the suborder Massarineae has estimated crown age around 104 Mya in scenario 1 (late Cretaceous), versus 130 Mya in scenario 2 (Cretaceous), the family Longipedicellataceae evolved around 44 Mya in scenario 1, versus 56 Mya in scenario 2 (Eocene). Although the estimates differ, they provide an idea of the order of evolutionary events and can thus be used to determine the order and rough times when different higher taxa evolved and can be used as evidence to support the status of these taxa ( $\mathrm{Li}$ et al. 2005, Beimforde et al. 2014, Hongsanan et al. 2016, Samarakoon et al. 2016, Zhao et al. 2016).

Previously dating studies estimated diversification events between Dothideomycetes and Arthoniomycetes to be over 300 million years ago in the Carboniferous period (Gueidan et al. 2011, Beimforde et al. 2014, McLaughlin \& Spatafora 2015, Pérez-Ortega et al. 2016). Our analysis demonstrate that applying a constraint at a node close to the tips is informative to the overall divergence times in the analysis. Consequently, the derived age estimates are reliant and affected by the selected fossil calibration point constraint in each analysis (Beimforde et al. 2014, Garnica et al. 2016). We present the scenario with oldest ages of ArthoniomycetesDothideomycetes split (scenario 2) which is more similar to previous studies.

\section{Evolution history of Longipedicellataceae}

Molecular phylogenetic analysis has been used to distinguish and provide evidence for various taxon levels in fungal systematics ( $\mathrm{Li}$ et al. 2005, Ertz et al. 2015, Maharachchikumbura et al. 2015). In this study, we applied the relaxed molecular clock to date divergences in Pleosporales. Based on our phylogenetic result, Massarineae and Plesporineae form two major clades in Pleosporales. In recent studies, 14 families and six taxa incertae sedis were placed in the suborder Massarineae (Tanaka et al. 2015, Li et al. 2016, Mapook et al. 2016). The split between Massarineae and Plesporineae occurred around 144 Mya. The Massarineae crown age is estimated to be around 130 Mya and the Plesporineae crown age is around 93 Mya. The values for Plesporineae may be underestimated since there may be basal taxa in this suborder that are not included in this study. Species of Pleosporineae are mostly reported as pathogens or endophytes in plants (Ginkel et al. 1998, Doilom et al. 2013, Ariyawansa et al. 2015). On the other hand, Massarineae are frequently saprobes in woody substrates (Barr 1979, Vijaykrishna et al. 2006, Kirk et al. 2008, Zhang et al. 2013, Tanaka et al. 2015). However, some taxa, such as Deniquelata (Didymosphaeriaceae), Setoseptoria (Lentitheciaceae) and Stagonospora (Massarinaceae) are plant pathogens (Ariyawansa et al. 2013, Hyde et al. 2016). Latoruaceae and Longipedicellataceae appear to have evolved around 75 Mya. Based on the molecular clock, Latoruaceae and Longipedicellataceae are warranted as distinct families within Massarineae.

The morphologies of these families correspond to the range found within Massarineae. Longipedicellata aptrootii was collected from submerged bamboo debris in different locations (Hyde \& Wong 1999, Zhang et al. 2016, this study). Pseudoxylomyces elegans was reported from various locations, but all from submerged wood in freshwater habitats (Goh et al. 1997, Prihatini et al. 2008, Tanaka et al. 2015). In our phylogenetic analysis, Longipedicellata aptrootii is closely related to Pseudoxylomyces elegans with high support (85\% ML/0.97 PP). This has been shown in 
previous studies with Pseudoxylomyces elegans forming a clade within Pleosporales (Prihatini et al. 2008, Tanaka et al. 2015). Morphologically, the genus Pseudoxylomyces, typified by P. elegans, is characterized by dematiaceous mycelia and gangliar-type of conidia production (Bhat 2010). Chlamydospore-like, thick-walled, terminal or intercalary, septate conidia structures seen in Longipedicellata aptrootii. Distinguishable conidia are absent in both species. In the phylogenetic analysis, Pseudoxylomyces along with Longipedicellata form a separate clade from Latoruaceae. Latoruaceae species were reported from various habitats, mostly from soil and some are thermotolerant (Agrawal \& Tiwari 1997, Sharma et al. 2015). Longipedicellataceae and Latoruaceae are similar in their hyphomycetes-like morphology of the asexual morph in Latorua, Matsushimamyces, and Polyschema. Nevertheless, those genera produce distinguishable conidia and not chlamydospore-like structures.

This study is unique in studies on Dothideomycetes as it uses a polyphasic approach in introducing a new family based on morphology, phylogeny and evolutionary divergence times and thus provides strong evidence for the application of Longipedicellataceae. It also provides estimates for the divergence of the subclass Pleosporomycetidae (249 Mya), order Pleosporales (211 Mya), suborders Massarineae (130 Mya) and Pleosporineae (93 Mya), families Parabambusicolaceae (81 Mya), Didymosphaeriaceae (66 Mya), Longipedicellataceae (56 Mya), Dictyosporiaceae (34 Mya) and the youngest family Macrodiplodiopsidaceae (6 Mya). Branch legth may represent unknown taxa that have either not been discovered or they are probably extinct taxa. Therefore, the taxon sampling in a group impacts on the age estimates of the crown nodes. It will be of considerable interest to compare the divergence times of the various higher taxon ranks (phylla, classes, orders, families, genera) across the kingdom fungi and establish if each ranks evolved at roughly the same time period or whether there is considerable disparity in the divergence times (Hedges et al. 2015, Samarakoon et al. 2016) and thus whether mycologists have treated different groups similarly or differently.

\section{Acknowledgements}

Chayanard Phukhamsakda would like to thank the Royal Golden Jubilee PhD Program under Thailand Research Fund, for the award of a scholarship no. PHD/0020/2557 to study towards a PhD. We thank the National Research Council of Thailand (grant for Dothideomycetes No: 58201020010) for supporting studies on Dothideomycetes. We would like to thank the Plant Germplasm and Genomics Center in Germplasm Bank of Wild Species, Kunming Institute of Botany for help with the molecular work. Chayanard would like to thank Professor Darbhe Jayarama Bhat for his valuable and suggestion on the description. Shaun Pennycook for checking and suggesting Latin names of the new taxa. Ausana Mapook, Jian Kui Liu, Anusha H. Ekanayaka thanked for their valuable suggestions. The authors extend their sincere appreciations to the Deanship of Scientific Research at King Saud University for its funding this Prolific Research group (PRG-1436-09).

\section{References}

Agrawal S, Tiwari SL. 1997 - Susceptibility level of few plants on basis of air pollution tolerance index. Indian Forester. 123, 319-322.

Ariyawansa HA, Hyde KD, Jayasiri SC, Buyck B et al. 2015 - Fungal diversity notes 111-252 Taxonomic and phylogenetic contributions to fungal taxa. Fungal Diversity 75, 27-274.

Ariyawansa HA, Maharachchikumbura SS, Karunarathne SC, Chukeatirote E et al. 2013 Deniquelata barringtoniae gen. et sp. nov., associated with leaf spots of Barringtonia asiatica. Phytotaxa 30, 11-20.

Barr ME. 1979 - A classification of Loculoascomycetes. Mycologia 71, 935-957.

Beimforde C, Feldberg K, Nylinder S, Rikkinen J et al. 2014 - Estimating the Phanerozoic history of the Ascomycota lineages: combining fossil and molecular data. Molecular Phylogenetics and Evolution 78, 386-398. 
Berbee M, Taylor JW. 2010 - Dating the molecular clock in fungi-how close are we?. Fungal Biology Reviews 24, 1-16.

Berbee ML, Taylor JW. 1993 - Dating the evolutionary radiations of the true fungi. Canadian Journal of Botany 71, 1114-1127.

Bhat J. 2010 - Fascinating microfungi (Hyphomycetes) of Western Ghats-India. Broadway Book Centre; Goa, India.

Boehm EW, Mugambi GK, Miller AN, Huhndorf SM et al. 2009 - A molecular phylogenetic reappraisal of the Hysteriaceae, Mytilinidiaceae and Gloniaceae (Pleosporomycetidae, Dothideomycetes) with keys to world species. Studies in Mycology 64, 49-83.

Boonmee S, D'souza MJ, Luo Z, Pinruan U et al. 2016 - Dictyosporiaceae fam. nov. Fungal Diversity 80, 457-482.

Campbell J, Shearer CA, Marvanová L. 2006 - Evolutionary relationships among aquatic anamorphs and teleomorphs: Lemonniera, Margaritispora, Goniopila. Mycological Research 110, 1025-1033.

Carbone I, Kohn LM. 1999 - A method for designing primer sets for speciation studies in filamentous ascomycetes. Mycologia 91, 553-556.

Chomnunti P, Hongsanan S, Hudson BA, Tian Q et al. 2014 - The sooty moulds. Fungal Diversity $66,1-36$.

Darriba D, Taboada GL, Doallo R, Posada D. 2012 - jModelTest 2: more models, new heuristics and parallel computing. Nature Methods 9, 772.

Doilom M, Liu JK, Jaklitsch WM, Ariyawansa H et al. 2013 - An outline of the family Cucurbitariaceae. Sydowia 65, 167-192.

Drummond AJ, Suchard MA, Xie D, Rambaut A. 2012 - Bayesian phylogenetics with BEAUti and the BEAST 1.7. Molecular Biology and Evolution 29, 1969-1973.

Ellis MB. 1971 - Dematiaceous hyphomycetes. Commonwealth Mycological Institute, Kew, Surrey, UK.

Ertz D, Diederich P, Lawrey JD, Berger F et al. 2015 - Phylogenetic insights resolve Dacampiaceae (Pleosporales) as polyphyletic: Didymocyrtis (Pleosporales, Phaeosphaeriaceae) with Phoma-like anamorphs resurrected and segregated from Polycoccum (Trypetheliales, Polycoccaceae fam. nov.). Fungal Diversity 74, 53-89.

Garnica S, Riess K, Schön ME, Oberwinkler F, Setaro SD. 2016 - Divergence times and phylogenetic patterns of Sebacinales, a highly diverse and widespread fungal lineage. PloS one $11, \mathrm{e} 0149531$.

Gernhard T, Hartmann K, Steel M. 2008 - Stochastic properties of generalised Yule models, with biodiversity applications. Journal of Mathematical Biology 253, 769-778.

Ginkel M, Rajaram S. 1998 - Breeding for resistance to spot blotch in wheat: Global perspective. Helminthosporium Blights of Wheat: Spot Blotch and Tan Spot. CIMMYT, Mexico DF, 162-169.

Goh TK, Ho WH, Hyde KD, Tsui KM. 1997 - Four new species of Xylomyces from submerged wood. Mycological Research 101, 1323-1328.

Gueidan C, Ruibal C, De Hoog GS, Schneider H. 2011 - Rock-inhabiting fungi originated during periods of dry climate in the late Devonian and middle Triassic. Fungal Biology 115, $987-$ 996.

Guindon S, Dufayard JF, Lefort V, Anisimova M et al. 2010 - New algorithms and methods to estimate maximum-likelihood phylogenies: assessing the performance of PhyML 3.0. Systematic Biology 59, 307-321.

Hall TA. 1999 - BioEdit: a user-friendly biological sequence alignment editor and analysis program for Windows 95/98/NT. Nucleic Acids Symposium Series 41, 95-98.

Hedges SB, Marin J, Suleski M, Paymer M, Kumar S. 2015 - Tree of life reveals clock-like speciation and diversification. Molecular Biology and Evolution 32, 835-845.

Hongsanan S, Sánchez-Ramírez S, Bhat DJ, Ariyawansa HA et al. 2016 - The evolution of fungal epiphytes. Mycosphere 7, 1690-1712. 
Huelsenbeck JP, Ronquist F. 2001 - MRBAYES: Bayesian inference of phylogenetic trees. Bioinformatics 17, 754-755.

Hyde KD, Hongsanan S, Jeewon R, Bhat DJ et al. 2016 - Fungal diversity notes 367-490: taxonomic and phylogenetic contributions to fungal taxa. Fungal Diversity 80, 1-270.

Hyde KD, Jones EBG, Liu JK, Ariyawansa HA et al. 2013 - Families of Dothideomycetes. Fungal Diversity 63, 1-313.

Hyde KD, Wong SW. 1999 - Didymella aptrootii sp. nov. from bamboo submerged in freshwater. Australasian Mycologist 18, 54-59.

Hyde KD. 1992 - Aigialus striatispora sp. nov. from intertidal mangrove wood. Mycological Research 96, 1044-1046.

Index Fungorum. 2016 - http://www.indexfungorum.org/Names/Names.asp. Accessed on 2016.

Jayasiri SC, Hyde KD, Ariyawansa HA, Bhat DJ et al. 2015 - The Faces of Fungi database: fungal names linked with morphology, phylogeny and human impacts. Fungal Diversity 74, 3-18.

Jones EG, Suetrong S, Sakayaroj J, Bahkali AH et al. 2015 - Classification of marine Ascomycota, Basidiomycota, Blastocladiomycota and Chytridiomycota. Fungal Diversity 73, 1-72.

Kirk PM, Cannon PF, Minter DW, Stalpers JA. 2008 - Dictionary of the Fungi. Tenth Edition CSIRO.

Kohlmeyer J, Schatz S. 1985 - Aigialus gen. nov. (Ascomycetes) with two new marine species from mangroves. Transactions of the British mycological Society 85, 699-707.

Kruys A, Wedin M. 2009 - Phylogenetic relationships and an assessment of traditionally used taxonomic characters in the Sporormiaceae (Pleosporales, Dothideomycetes, Ascomycota), utilising multi-gene phylogenies. Systematics and Biodiversity 7, 465-478.

Li GJ, Hyde KD, Zhao RN, Hongsanan S et al. 2016 - Fungal diversity notes 367-490: taxonomic and phylogenetic contributions to fungal taxa. Fungal Diversity 78, 1-237.

Li Y, Hyde KD, Jeewon R, Cai L. et al. 2005 - Phylogenetics and evolution of nematode-trapping fungi (Orbiliales) estimated from nuclear and protein coding genes. Mycologia. 97, 10341046.

Liu JK, Phookamsak R, Dai DQ, Tanaka K et al. 2014 - Roussoellaceae, a new pleosporalean family to accommodate the genera Neoroussoella gen. nov., Roussoella and Roussoellopsis. Phytotaxa 181, 1-33.

Lumbsch HT, Huhndorf SM. 2010 -Outline of Ascomycota-Part two. Notes on ascomycete systematics. Nos. 4751-5113. Fieldiana Life and Earth Sciences 1, 1-64.

Maharachchikumbura SSN, Hyde KD, Jones EBG, McKenzie EHC et al. 2015 - Towards a natural classification and backbone tree for Sordariomycetes. Fungal Diversity 72, 199-301.

Mapook A, Boonmee S, Liu JK, Jones EBG et al. 2016 - Taxonomic and phylogenetic placement of Phaeodimeriella (Pseudoperisporiaceae, Pleosporales). Cryptogamie Mycologie 37, 120.

McLaughlin D, Spatafora JW. 2015 - Systematics and evolution (Eds.) The Mycota. SpringerVerlag Berlin Heidelberg, 1-311.

Mindell RA, Stockey RA, Beard G, Currah RS. 2007 - Margaretbarromyces dictyosporus gen. sp. nov.: a permineralized corticolous ascomycete from the Eocene of Vancouver Island, British Columbia. Mycological Research 111, 680-684.

Mugambi GK, Huhndorf SM. 2009 - Molecular phylogenetics of Pleosporales: Melanommataceae and Lophiostomataceae re-circumscribed (Pleosporomycetidae, Dothideomycetes, Ascomycota). Studies in Mycology 64, 103-121.

Nylander JAA. 2004 - MrModeltest v2. Program distributed by the author. Evolutionary Biology Centre, Uppsala University.

O'Meara BC, Ané C, Sanderson MJ, Wainwright PC. 2006 - Testing for different rates of continuous trait evolution using likelihood. Evolution 60, 922-933.

Pérez-Ortega S, Garrido-Benavent I, Grube M, Olmo R, de los Ríos A. 2016 - Hidden diversity of marine borderline lichens and a new order of fungi: Collemopsidiales (Dothideomyceta). Fungal Diversity 80, 285-300. 
Phukhamsakda C, Ariyawansa HA, Phookamsak R, Chomnunti P et al. 2015 - Muriphaeosphaeria galatellae gen. et sp. nov. in Phaeosphaeriaceae (Pleosporales). Phytotaxa 227, 55-65.

Prieto M, Wedin M. 2013 - Dating the diversification of the major lineages of Ascomycota (Fungi). PLoS One 14, e65576.

Prihatini R, Boonyuen N, Sivichai S. 2010 - Phylogenetic evidence that two submerged-habitat fungal species, Speiropsis pedatospora and Xylomyces chlamydosporus, belong to the order Jahnulales insertae sedis Dothideomycetes. Microbiology Indonesia 2, 136-140.

Rambaut A, Drummond A. 2008 - FigTree: Tree figure drawing tool, version 1.2. 2. Institute of Evolutionary Biology, University of Edinburgh.

Rambaut A, Suchard MA, Xie D, Drummond AJ. 2014 - Tracer v1.6, Available from http://beast.bio.ed.ac.uk/Tracer.

Samarakoon MC, Hyde KD, Promputtha I, Ariyawansa HA, Hongsanan S. 2016. Divergence and ranking of taxa across the kingdoms Animalia, Fungi and Plantae. Mycosphere 7, 16781689.

Schmidt AR, Perrichot V, Svojtka M, Anderson KB et al. 2010 - Cretaceous African life captured in amber. Proceedings of the National Academy of Sciences 107, 7329-7334.

Schmit JP, Shearer CA. 2003 - A checklist of mangrove-associated fungi, their geographical distribution and known host plants. Mycotaxon 85, 423-477.

Schoch CL, Crous PW, Groenewald JZ, Boehm EWA et al. 2009 - A class-wide phylogenetic assessment of Dothideomycetes. Studies in Mycology 64, 1-15.

Seifert KA, Morgan-Jones G, Gams W, Kendrick B. 2011 - The Genera of Hyphomycetes. CBS Biodiversity Series 9, 1-997.

Sharma R, Sharma R, Crous PW. 2015 - Matsushimamyces, a new genus of keratinophilic fungi from soil in central India. International Mycological Association fungus 6, 337-343.

Silvestro D, Michalak I. 2012 - raxmlGUI: a graphical front-end for RAxML. Organisms Diversity \& Evolution 12, 335-337.

Stamatakis A, Hoover P, Rougemont J. 2008 - A rapid bootstrap algorithm for the RAxML web servers. Systematic biology 57, 758-771.

Stamatakis A. 2014 - RAxML Version 8: A tool for Phylogenetic Analysis and Post-Analysis of Large Phylogenies. Bioinformatics 30, 1312-1313.

Suetrong S, Schoch CL, Spatafora JW, Kohlmeyer J et al. 2009 - Molecular systematics of the marine Dothideomycetes. Studies in Mycology 64, 155-73.

Tamura K, Stecher G, Peterson D, Filipski A, Kumar S. 2013 - MEGA6: Molecular Evolutionary Genetics Analysis Version 6.0. Molecular Biology and Evolution 30, 2725-2729.

Tanaka K, Hirayama K, Yonezawa H, Sato G et al. 2015 - Revision of the Massarineae (Pleosporales, Dothideomycetes). Studies in Mycology 82, 75-136.

Taylor EL, Taylor TN, Krings M. 2009 - Fungi, Bacteria, and Lichens, In Paleobotany: the biology and evolution of fossil plants (pp. 71-119). Academic Press: San Diego, CA, USA.

Taylor JW, Berbee ML. 2006 - Dating divergences in the Fungal Tree of Life: review and new analyses. Mycologia 98, 838-49.

Taylor TN, Krings M, Taylor EL. 2015 - Ascomycota, In Fossil fungi (pp. 129-171). Academic Press: San Diego, CA, USA.

Vijaykrishna D, Jeewon R, Hyde KD. 2006 - Molecular taxonomy, origins and evolution of freshwater ascomycetes. Fungal Diversity 23, 351-390.

Vilgalys R, Hester M. 1990 - Rapid genetic identification and mapping of enzymatically amplified ribosomal DNA from several Cryptococcus species. Journal of Bacteriology 17, 4238-4246.

White TJ, Bruns T, Lee S, Taylor J. 1990 - Amplification and direct sequencing of fungal ribosomal RNA genes for phylogenetics. PCR Protocols: A guide to Methods and Applications 18, 315-322.

Wijayawardene NN, Crous PW, Kirk PM, Hawksworth DL et al. 2014 - Naming and outline of Dothideomycetes-2014 including proposals for the protection or suppression of generic names. Fungal Diversity 69, 1-55. 
Zhang H, Dong W, Hyde KD, Bahkali AH et al. 2016 - Molecular data shows Didymella aptrootii is a new genus in Bambusicolaceae. Phytotaxa 247, 99-108.

Zhang H, Hyde KD, Abdel-Wahab MA, Abdel-Aziz FA, et al. 2013 - A modern concept for Helicascus with a Pleurophomopsis-like asexual state. Sydowia, 65, 147-166.

Zhang Y, Crous PW, Schoch CL, Hyde KD. 2012 - Pleosporales. Fungal Diversity 53, 1-221.

Zhang Y, Wang HK, Fournier J, Crous PW et al. 2009 - Towards a phylogenetic clarification of Lophiostoma/Massarina and morphologically similar genera in the Pleosporales. Fungal Diversity 53, 225-251.

Zhao R, Zhou J, Chen J, Margaritescu S et al. 2016 - Towards standardizing taxonomic ranks using divergence times - A case study for reconstruction of the Agaricus taxonomic system. Fungal Diversity 78, 239-292.

Zhaxybayeva O, Gogarten JP. 2002 - Bootstrap, Bayesian probability and maximum likelihood mapping: exploring new tools for comparative genome analyses. BMC Genomics 3, 4. 